\title{
Obesity affects taste receptor expression in the brain
}

Taste perception plays a well-known role in food consumption: a bite of something sweet or savory often encourages food intake, while a bitter sensation often induces the opposite. Although taste perception is typically associated with peripheral receptors in the mouth and tongue, taste receptor genes are also expressed in various extra-oral tissues like the brain and gut. Researchers are considering whether these receptor genes may play a broader role in food intake, gastric functions, and energy homeostasis. If true, this system may be critical to understanding normal appetitive behaviors and controlling weightrelated disorders.

In a recent study, Herrera Moro Chao et al. (Sci. Rep. 6, 29094; 2016) examined how obesity and fasting affect gene expression of taste receptors and associated signaling complexes, focusing on several brain regions in mice. Using in situ hybridization and quantitative PCR, the authors first verified the extra-oral expression of sweet and bitter

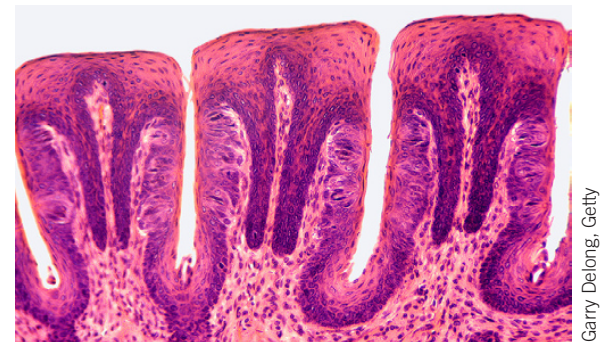

taste receptor genes ( $G$ protein-coupled taste receptors, GPCTRs) along with their associated downstream signaling subunits. Multiple areas expressed GPCTRs, including the brainstem and hypothalamus, which are collectively involved in energy homeostasis.

The researchers used lean, obese, and fasted mice to examine how these nutritional statuses affect the expression of extraoral GPCTRs. Lean mice that were fasted for 16 hours displayed lower levels of bitter GPCTRs and associated signaling molecules in the brainstem but not the hypothalamus, and displayed no differences in sweet-tasting receptor genes. However, significant differences in expression were observed when comparing obese and lean mice: overall, obese mice displayed lower expression of sweet and bitter taste receptor genes than lean mice, in both the hypothalamus and brainstem. This suggests that short-term changes in nutritional status, like fasting, do not exert major effects on GPCTR expression; meanwhile, chronic changes like obesity do appear to affect taste receptor expression in brain regions involved with metabolism.

The authors propose that the observed downregulation of sweet taste receptor genes in the brains of obese mice may promote obesity by causing the animals to desire a largerthan-normal amount of taste sensation to achieve sufficient taste perception. Further research into this hypothesized mechanism could yield new treatments for obesity, as well as a better understanding of how nutrition interacts with metabolism.

James E. Niemeyer 